\title{
ON ONE MATHEMATICAL MODEL DESCRIBED BY BOUNDARY VALUE PROBLEM FOR THE BIHARMONIC EQUATION
}

\author{
V.V. Karachik, South Ural State University, Chelyabinsk, Russian Federation, \\ karachik@susu.ru, \\ B.T. Torebek, Institute of Mathematics and Mathematical Modelling, Almaty, \\ Kazakhistan, torebek@math.kz
}

In this paper mathematical model described by a generalized third boundary value problem for the homogeneous biharmonic equation in the unit ball with boundary operators up to the third order containing normal derivatives and Laplacian is investigated. Particular cases of the considered mathematical model are the classical models described by Dirichlet, Riquier, and Robin problems, and the Steklov spectral problem, as well as many other mathematical models generated by these boundary conditions. Two existence theorems for the solution of the problem are proved. Existence conditions are obtained in the form of orthogonality on the boundary of some linear combination of boundary functions to homogeneous harmonic polynomials of a particular order. The obtained results are illustrated by some special cases of the general problem.

Keywords: mathematical model; biharmonic equation; boundary value problems; Laplace operator.

\section{Introduction}

A significant number of mathematical models in physics and engineering lead to partial differential equations. The steady processes of various physical nature are described by the partial differential equations of elliptic type. One of the important special cases of fourth order elliptic equations is the biharmonic equation $\Delta^{2} u(x)=f(x)$.

Investigation of mathematical models of problems of the plane deformation of the elasticity theory in many cases is reduced to integration of the biharmonic equation with the appropriate boundary conditions and under some uniqueness conditions for the unknown function.

Moreover investigation of many mathematical models of continuum mechanics are reduced to solving the harmonic and biharmonic equations. However the convenient analytical expressions for the solutions are obtained only for the certain domains of particular forms. Application of biharmonic problems in mathematical models of mechanics and physics can be found in the numerous scientific investigations (see, for example, [1-3]).

Multiple applications of boundary value problems for the biharmonic equation in mathematical models of mechanics and physics encourage investigation of various formulations of boundary value problems for the biharmonic equation. The class of biharmonic functions includes a class of harmonic functions and is a subclass of polyharmonic functions. One of the first important works on the biharmonic equation is the article by Almansi [4]. Following this work, there was a big interest in studying of boundary value problems for the biharmonic equation (see [5-7]).

The Dirichlet problem (see [8]) is a well known boundary value problem for biharmonic equation. In recent years other types of boundary value problems for the biharmonic 
equation, such as the problems by Riquier (see [9]), by Neumann (see [10-12]), by Robin and etc are actively studied. In the spectral theory the Steklov spectral problem is of some interest.

The present paper is organized as follows. In Section 1 for the biharmonic equation in the unit ball a boundary value problem (1) - (2) with the boundary conditions of general type, called by us as a generalized third boundary value problem is formulated. Particular cases of the problem are considered in Section 4. In Section 2, namely in Theorem 2, the conditions of unconditional solvability of the problem for the homogeneous biharmonic equation are found. These conditions coincide with the conditions of uniqueness from Theorem 1. In Theorem 3 of Section 3 the case, when the conditions of Theorem 2 are not fulfilled, but a solution of the considered problem still exists, are obtained.

\section{Statement of a Problem}

Let $S=\left\{x \in \mathbb{R}^{n}:|x|<1\right\}$ be an $n$-dimensional unit ball in the Euclidean space $\mathbb{R}^{n}$ with the norm $|x|=\sqrt{x_{1}^{2}+x_{2}^{2}+\ldots+x_{n}^{2}}$, and $\partial S=\left\{x \in \mathbb{R}^{n}:|x|=1\right\}$ be an unit sphere. In $S$ we consider the following boundary value problem for the biharmonic equation

$$
\begin{gathered}
\Delta^{2} u=f(x), \quad x \in S, \\
a_{00} u+a_{01} \frac{\partial}{\partial \nu} u+\left.a_{02} \Delta u\right|_{\partial S}=\varphi_{1}(s), \quad s \in \partial S \\
a_{11} \frac{\partial}{\partial \nu} u+a_{12} \Delta u+\left.a_{13} \frac{\partial}{\partial \nu} \Delta u\right|_{\partial S}=\varphi_{2}(s), \quad s \in \partial S,
\end{gathered}
$$

where $\frac{\partial}{\partial \nu}$ is the outside normal derivative, $a_{0 j}$ and $a_{1 j}$ where $j=1,2,3$ are real constants, and $f(x), \varphi_{1}(x), \varphi_{2}(x)$ are defined function, smoothness of which will be given below. As a solution of problem (1) - (2) we consider a biharmonic in $S$ function $u(x)$ from the class $u \in C^{4}(S) \cap C^{3}(\bar{S})$ which satisfies on $\partial S$ conditions (2).

Problem $(1)-(2)$ generalizes the known Dirichlet problem $\left(a_{00} \neq 0, a_{11} \neq 0\right.$, and all other coefficients are zero), Riquier problem $\left(a_{00} \neq 0, a_{12} \neq 0\right.$, and all other coefficients are zero), but does not generalize the Neumann boundary value problem. If $a_{00} \neq 0, a_{12}>0$, $a_{11}<0$, and all other coefficients vanish, then conditions (2) coincide with the Steklov conditions [13]. In [14] uniqueness of a solution for (1) - (2) was proved.

Theorem 1. Solution of problem (1) - (2) is unique if and only if the following polynomial

$$
\Delta(\lambda)=\left|\begin{array}{cc}
a_{00}+a_{01} \lambda & 2 a_{01}+(2 n+4 \lambda) a_{02} \\
\lambda a_{11} & 2 a_{11}+(2 n+4 \lambda) a_{12}+\lambda(2 n+4 \lambda) a_{13}
\end{array}\right|
$$

has no roots in $\mathbb{N}_{0}=\mathbb{N} \cup\{0\}$. If $\Delta(m)=0$, then the homogeneous problem (1) - (2) has a solution

$$
u(x)=\left(C_{2}|x|^{2}+C_{1}-C_{2}\right) H_{m}(x),
$$

where $H_{m}(x)$ is an arbitrary homogeneous harmonic polynomial of degree $m$, and the constants $C_{1}, C_{2}$ are found from the system of algebraic equations

$$
\left(\begin{array}{cc}
a_{00}+m a_{01} & 2 a_{01}+(2 n+4 m) a_{02} \\
m a_{11} & 2 a_{11}+(2 n+4 m) a_{12}+m(2 n+4 m) a_{13}
\end{array}\right)\left(\begin{array}{c}
C_{1} \\
C_{2}
\end{array}\right)=0 .
$$




\section{Unconditional Existence of a Solution}

One auxiliary assertion in which harmonic functions from the Almansi representation are explicitly defined can be easily proved.

Lemma 1. Let $u(x)$ be a biharmonic function in $S$, then the following functions

$$
u_{0}(x)=u(x)-\frac{|x|^{2}}{2} \int_{0}^{1} t^{n-1} \Delta u\left(t^{2} x\right) d t, \quad u_{1}(x)=\frac{1}{2} \int_{0}^{1} t^{n-1} \Delta u\left(t^{2} x\right) d t
$$

are harmonic in $S$ and such that the Almansi representation $u(x)=u_{0}(x)+|x|^{2} u_{1}(x)$ holds in $S$.

Theorem 2. Solution of the problem

$$
\begin{gathered}
\Delta^{2} u=0, \quad x \in S, \\
a_{00} u+a_{01} \frac{\partial}{\partial \nu} u+\left.a_{02} \Delta u\right|_{\partial S}=\varphi_{1}(s), \quad x \in \partial S, \\
a_{11} \frac{\partial}{\partial \nu} u+a_{12} \Delta u+\left.a_{13} \frac{\partial}{\partial \nu} \Delta u\right|_{\partial S}=\varphi_{2}(s), \quad x \in \partial S,
\end{gathered}
$$

from the class $u \in C^{3}(\bar{S})$ for arbitrary functions $\varphi_{1} \in C^{2}(\partial S)$ and $\varphi_{2} \in C^{1}(\partial S)$ exist, if and only if the polynomial

$$
\Delta(\lambda)=\left|\begin{array}{cc}
a_{00}+a_{01} \lambda & 2 a_{01}+(2 n+4 \lambda) a_{02} \\
a_{11} \lambda & 2 a_{11}+(2 n+4 \lambda) a_{12}+\lambda(2 n+4 \lambda) a_{13}
\end{array}\right|
$$

has no roots in $\mathbb{N}_{0}$.

Proof. Consider the harmonic in $S$ functions $v_{1}(x)$ and $v_{2}(x)$ such that for them the equalities

$$
\left.v_{1}(x)\right|_{\partial S}=\varphi_{1}(s), \quad s \in \partial S,\left.\quad v_{2}(x)\right|_{\partial S}=\varphi_{2}(s), \quad s \in \partial S
$$

hold, i.e. $v_{1}(x)$ and $v_{2}(x)$ are solutions of the Dirichlet problem for the Laplace equation in $S$. By Lemma 1 for the biharmonic function $u(x)$ the Almansi representation holds. Let us find the functions $u_{0}(x)$ and $u_{1}(x)$ under which the biharmonic function $u(x)=u_{0}(x)+|x|^{2} u_{1}(x)$ is a solution of $(5)-(6)$. Consider the operators $L_{1}$ and $L_{2}$ from the Theorem 1. Then, because of equality

$$
\Delta\left(|x|^{2} v(x)\right)=(2 n+4 \Lambda) v(x)
$$

we have

$$
\begin{aligned}
& L_{1} u=L_{1}\left(u_{0}(x)+|x|^{2} u_{1}(x)\right)=a_{00} u+a_{01} \Lambda u+a_{02}(2 n+4 \Lambda) u_{1}, \\
& L_{2} u=L_{2}\left(u_{0}(x)+|x|^{2} u_{1}(x)\right)=a_{11} \Lambda u+a_{12}(2 n+4 \Lambda) u_{1}+a_{13} \Lambda(2 n+4 \Lambda) u_{1} .
\end{aligned}
$$

Taking the limit as $x \rightarrow s \in \partial S$, as in Theorem 1, we obtain

$$
\begin{aligned}
& a_{00}\left(u_{0}+u_{1}\right)+a_{01}\left(\Lambda u_{0}+(\Lambda+2) u_{1}\right)+a_{02}(2 n+4 \Lambda) u_{1}=\varphi_{1}(s) \\
& a_{11}\left(\Lambda u_{0}+(\Lambda+2) u_{1}\right)+a_{12}(2 n+4 \Lambda) u_{1}+a_{13} \Lambda(2 n+4 \Lambda) u_{1}=\varphi_{2}(s) .
\end{aligned}
$$

By the uniqueness of the Dirichlet problem solution for the Laplace equation in $S$, we conclude that equalities (7) can be extended to $S$ 


$$
\begin{aligned}
& a_{00}\left(u_{0}+u_{1}\right)+a_{01}\left(\Lambda u_{0}+(\Lambda+2) u_{1}\right)+a_{02}(2 n+4 \Lambda) u_{1}=v_{1}(x), \\
& a_{11}\left(\Lambda u_{0}+(\Lambda+2) u_{1}\right)+a_{12}(2 n+4 \Lambda) u_{1}+a_{13} \Lambda(2 n+4 \Lambda) u_{1}=v_{2}(x),
\end{aligned}
$$

because by the properties of the operator $\Lambda$ the functions on the left-hand side in the last equalities are harmonic in $S$. We rewrite the resulting equations in the matrix form

$$
\left(\begin{array}{cc}
a_{00}+a_{01} \Lambda & a_{00}+(\Lambda+2) a_{01}+(2 n+4 \Lambda) a_{02} \\
a_{11} \Lambda & (\Lambda+2) a_{11}+(2 n+4 \Lambda)\left(a_{12}+\Lambda a_{13}\right)
\end{array}\right)\left(\begin{array}{l}
u_{0} \\
u_{1}
\end{array}\right)=\left(\begin{array}{l}
v_{1} \\
v_{2}
\end{array}\right) .
$$

Equation (8) is a system of differential equations in harmonic functions in $S$. Let us show that (8) holds also in $\bar{S}$. By the theorem's condition we have $u(x) \in C^{3}(\bar{S})$, then according to formulas (4) we obtain $u_{0}(x), u_{1}(x) \in C^{1}(\bar{S})$. Taking into account that $\Lambda u_{1}=\frac{1}{4} \Delta u-\frac{n}{2} u_{1}$ (see Lemma 1) we obtain $\Lambda u_{1}(x) \in C^{1}(\bar{S})$. However, because $u_{0}(x) \in C^{1}(\bar{S})$ and $\Lambda u_{1}(x) \in C^{1}(\bar{S})$ equation (8) is fulfilled in $\bar{S}$. So, from the above reasoning we can conclude that if the function $u(x)=u_{0}(x)+|x|^{2} u_{1}(x) \in C^{3}(\bar{S})$ is a solution of the problem (5) - (6), then harmonic functions $u_{0}(x)$ and $u_{1}(x)$ satisfy $(8)$ in $\bar{S}$.

Converse assertion also holds, i.e. if the harmonic functions $u_{0}(x) \in C^{1}(\bar{S})$ and $u_{1}(x) \in C^{1}(\bar{S}), \Lambda u_{1}(x) \in C^{1}(\bar{S})$ satisfy (8) in $\bar{S}$, then they satisfy (7), and therefore the function $u(x)=u_{0}(x)+|x|^{2} u_{1}(x)$ is a solution of $(5)-(6)$. Since $\Delta u(x)=(2 n+4 \Lambda) u_{1}(x)$, then $u(x) \in C^{3}(\bar{S})$. Thus, problem $(5)-(6)$ and equation (8) in $\bar{S}$ are equivalent.

Consider the following matrix

$$
A(\lambda)=\left(\begin{array}{cc}
a_{00}+a_{01} \lambda & a_{00}+(\lambda+2) a_{01}+(2 n+4 \lambda) a_{02} \\
a_{11} \lambda & (\lambda+2) a_{11}+(2 n+4 \lambda) a_{12}+\lambda(2 n+4 \lambda) a_{13}
\end{array}\right)
$$

depending on a parameter $\lambda \in \mathbb{R}$. It is easy to see that $\operatorname{det} A(\lambda)=\Delta(\lambda)$ and $\operatorname{deg} \operatorname{det} A(\lambda)=3$. Therefore, under the conditions of the theorem the matrix $A(\lambda)$ is nonsingular for $\lambda \in \mathbb{N}_{0}$. System (8) can be written in the form

$$
A(\Lambda) U(x)=V(x), \quad x \in \bar{S},
$$

where $U=\left(\begin{array}{l}u_{0} \\ u_{1}\end{array}\right)$ and $V=\left(\begin{array}{l}v_{1} \\ v_{2}\end{array}\right)$. We expand the polynomial $\operatorname{det} A(\lambda)$

$$
\operatorname{det} A(\lambda)=\Delta(\lambda)=c\left(\lambda-\lambda_{1}\right)\left(\lambda-\lambda_{2}\right)\left(\lambda-\lambda_{3}\right),
$$

where $\lambda_{i} \in \mathbb{C}, i=1,2,3$ are the roots of the polynomial $\Delta(\lambda)$.

1) Let all the roots $\lambda_{i}$ of the polynomial $\Delta(\lambda)$ be different. It is well known that in this case

$$
\frac{1}{\Delta(\lambda)}=\frac{b_{1}}{\lambda-\lambda_{1}}+\frac{b_{2}}{\lambda-\lambda_{2}}+\frac{b_{3}}{\lambda-\lambda_{3}}
$$

where $b_{i} \in \mathbb{R}$. The inverse matrix $A^{-1}(\lambda)$ to $A(\lambda)$ has the form $A^{-1}(\lambda)=\frac{1}{\Delta(\lambda)} A^{*}(\lambda)$, where

$$
A^{*}(\lambda)=\left(\begin{array}{cc}
(\lambda+2) a_{11}+(2 n+4 \lambda)\left(a_{12}+\lambda a_{13}\right) & -a_{00}-(\lambda+2) a_{01}-(2 n+4 \lambda) a_{02} \\
-a_{11} \lambda & a_{00}+a_{01} \lambda
\end{array}\right) .
$$

Consider the following operators depending on the roots $\lambda_{i}$ (see [16])

$$
M_{i}(\Lambda) v(x)=\int_{0}^{1} t^{-\lambda_{i}-1} v(t x) d t,
$$


where $i=1,2,3$. If $\lim _{t \rightarrow 0} t^{-\lambda_{i}} w(t x)=0$, then it is easy to see that for a differentiable function $w(x)$ the following equalities hold

$$
\begin{gathered}
\left(\Lambda-\lambda_{i}\right) M_{i}(\Lambda) w=\left(\Lambda-\lambda_{i}\right) \int_{0}^{1} t^{-\lambda_{i}-1} w(t x) d t=-\lambda_{i} \int_{0}^{1} t^{-\lambda_{i}-1} w(t x) d t+ \\
+\int_{0}^{1} t^{-\lambda_{i}} \sum_{i=1}^{n} x_{i} w_{x_{i}}(t x) d t=-\lambda_{i} \int_{0}^{1} t^{-\lambda_{i}-1} w(t x) d t+\int_{0}^{1} t^{-\lambda_{i}} w_{t}(t x) d t= \\
-\lambda_{i} \int_{0}^{1} t^{-\lambda_{i}-1} w(t x) d t+\left.t^{-\lambda_{i}} w(t x)\right|_{0} ^{1}+\lambda_{i} \int_{0}^{1} t^{-\lambda_{i}-1} w(t x) d t=\left.t^{-\lambda_{i}} w(t x)\right|_{0} ^{1}=w(x) .
\end{gathered}
$$

Since the following equalities are true

$$
\begin{gathered}
1=\Delta(\lambda)\left(\frac{b_{1}}{\lambda-\lambda_{1}}+\frac{b_{2}}{\lambda-\lambda_{2}}+\frac{b_{3}}{\lambda-\lambda_{3}}\right)=\frac{b_{1} \Delta(\lambda)}{\lambda-\lambda_{1}}+\frac{b_{2} \Delta(\lambda)}{\lambda-\lambda_{2}}+\frac{b_{3} \Delta(\lambda)}{\lambda-\lambda_{3}}= \\
=c b_{1}\left(\lambda-\lambda_{2}\right)\left(\lambda-\lambda_{3}\right)+c b_{2}\left(\lambda-\lambda_{1}\right)\left(\lambda-\lambda_{3}\right)+c b_{3}\left(\lambda-\lambda_{1}\right)\left(\lambda-\lambda_{2}\right),
\end{gathered}
$$

then for the function $w \in C^{2}(\bar{S})$ such that $\lim _{t \rightarrow 0} t^{-\lambda_{i}} w(t x)=0, \quad i=1,2,3$ we have

$$
\begin{aligned}
\Delta(\Lambda)\left(b_{1} M_{1}(\Lambda)+b_{2} M_{2}(\Lambda)\right. & \left.+b_{3} M_{3}(\Lambda)\right) w=\left(c b_{1}\left(\Lambda-\lambda_{2}\right)\left(\Lambda-\lambda_{3}\right)+\right. \\
& \left.+c b_{2}\left(\Lambda-\lambda_{1}\right)\left(\Lambda-\lambda_{3}\right)+c b_{3}\left(\Lambda-\lambda_{1}\right)\left(\Lambda-\lambda_{2}\right)\right) w=1 \cdot w .
\end{aligned}
$$

Therefore, if the function $V(x)$ satisfies the conditions $\lim _{t \rightarrow 0} t^{-\lambda_{i}} V(t x)=0$, where $i=$ $1,2,3$, then the solution of (9) can be written in the form

$$
U(x)=A^{*}(\Lambda)\left(b_{1} M_{1}(\Lambda)+b_{2} M_{2}(\Lambda)+b_{3} M_{3}(\Lambda)\right) V(x) .
$$
$\bar{S}$, i.e.

Indeed, the function $U(x)$, which is found from (12) by virtue of (11) satisfies (9) in

$$
\begin{gathered}
A(\Lambda) U(x)=A(\Lambda) A^{*}(\Lambda)\left(b_{1} M_{1}(\Lambda)+b_{2} M_{2}(\Lambda)+b_{3} M_{3}(\Lambda)\right) V(x)= \\
=\Delta(\Lambda)\left(b_{1} M_{1}(\Lambda)+b_{2} M_{2}(\Lambda)+b_{3} M_{3}(\Lambda)\right) V(x)=V(x) .
\end{gathered}
$$

2) Let two roots of the polynomial $\Delta(\lambda)$ be equal $\lambda_{1}=\lambda_{2}$, i.e. $\Delta(\lambda)=c\left(\lambda-\lambda_{1}\right)^{2}(\lambda-$ $\left.\lambda_{3}\right)$. It is known that in this case $\frac{1}{\Delta(\lambda)}=\frac{b_{1}}{\lambda-\lambda_{1}}+\frac{b_{2}}{\left(\lambda-\lambda_{1}\right)^{2}}+\frac{b_{3}}{\lambda-\lambda_{3}}$, and hence

$$
\begin{aligned}
1=\Delta(\lambda)\left(\frac{b_{1}}{\lambda-\lambda_{1}}+\frac{b_{2}}{\left(\lambda-\lambda_{1}\right)^{2}}\right. & \left.+\frac{b_{3}}{\lambda-\lambda_{3}}\right)=\frac{b_{1} \Delta(\lambda)}{\lambda-\lambda_{1}}+\frac{b_{2} \Delta(\lambda)}{\left(\lambda-\lambda_{1}\right)^{2}}+\frac{b_{3} \Delta(\lambda)}{\lambda-\lambda_{3}}= \\
& =c b_{1}\left(\lambda-\lambda_{1}\right)\left(\lambda-\lambda_{3}\right)+c b_{2}\left(\lambda-\lambda_{3}\right)+c b_{3}\left(\lambda-\lambda_{1}\right)^{2} .
\end{aligned}
$$

Consider the operator

$$
M_{1}^{(2)}(\Lambda) v(x)=-\int_{0}^{1} \ln t t^{-\lambda_{1}-1} v(t x) d t
$$

It is easy to see that if the function $w(x)$ is differentiable, then the equalities

$$
\left(\Lambda-\lambda_{1}\right) M_{1}^{(2)}(\Lambda) w=M_{1}(\Lambda) w+\left.\ln t t^{-\lambda_{1}} w(t x)\right|_{0} ^{1}=M_{1}(\Lambda) w
$$


hold and hence $\left(\Lambda-\lambda_{1}\right)^{2} M_{1}^{(2)}(\Lambda) w=w$, if $\lim _{t \rightarrow 0} \ln t t^{-\lambda_{1}} w(t x)=0$. Due to (13) a function $w \in C^{2}(\bar{S})$ such that $\lim _{t \rightarrow 0} \ln t t^{-\lambda_{1}} w(t x)=0$ satisfies the equality

$$
\begin{gathered}
\Delta(\Lambda)\left(b_{1} M_{1}(\Lambda)+b_{2} M_{1}^{(2)}(\Lambda)+b_{3} M_{3}(\Lambda)\right) w= \\
=\left(c b_{1}\left(\Lambda-\lambda_{1}\right)\left(\Lambda-\lambda_{3}\right)+c b_{2}\left(\Lambda-\lambda_{3}\right)+c b_{3}\left(\Lambda-\lambda_{1}\right)^{2}\right) w=1 \cdot w .
\end{gathered}
$$

So, if $\lambda_{1}=\lambda_{2}$ and the function $V(x)$ satisfies the conditions $\lim _{t \rightarrow 0} \ln t t^{-\lambda_{1}} V(t x)=0$ and $\lim _{t \rightarrow 0} t^{-\lambda_{3}} V(t x)=0$, then the solution of (9) can be written as

$$
U(x)=A^{*}(\Lambda)\left(b_{1} M_{1}(\Lambda)+b_{2} M_{1}^{(2)}(\Lambda)+b_{3} M_{3}(\Lambda)\right) V(x) .
$$

3) Let the three roots of the polynomial $\Delta(\lambda)$ be equal, i.e. $\Delta(\lambda)=c\left(\lambda-\lambda_{1}\right)^{3}$. We consider the operator

$$
M_{1}^{(3)}(\Lambda) v(x)=\frac{1}{2} \int_{0}^{1} \ln ^{2} t t^{-\lambda_{1}-1} v(t x) d t .
$$

It is easy to see that if the function $w(x)$ is a differentiable one, then we have

$$
\begin{gathered}
\left(\Lambda-\lambda_{1}\right) M_{1}^{(3)}(\Lambda) w=\left(\Lambda-\lambda_{1}\right) \frac{1}{2} \int_{0}^{1} \ln ^{2} t t^{-\lambda_{1}-1} w(t x) d t= \\
=-\frac{\lambda_{1}}{2} \int_{0}^{1} \ln ^{2} t t^{-\lambda_{1}-1} w(t x) d t+\frac{1}{2} \int_{0}^{1} \ln ^{2} t t^{-\lambda_{1}} w_{t}(t x) d t= \\
=-\frac{\lambda_{1}}{2} \int_{0}^{1} \ln ^{2} t t^{-\lambda_{1}-1} w(t x) d t+\left.\frac{1}{2} \ln ^{2} t t^{-\lambda_{1}} w(t x)\right|_{0} ^{1}+\frac{\lambda_{1}}{2} \int_{0}^{1} \ln ^{2} t t^{-\lambda_{1}-1} w(t x) d t- \\
-\int_{0}^{1} \ln t t^{-\lambda_{1}-1} w(t x) d t=M_{1}^{(2)}(\Lambda) w+\left.\frac{1}{2} \ln ^{2} t t^{-\lambda_{1}} w(t x)\right|_{0} ^{1}=M_{1}^{(2)}(\Lambda) w,
\end{gathered}
$$

provided that $\lim _{t \rightarrow 0} \ln ^{2} t t^{-\lambda_{1}} w(t x)=0$. By the above $\left(\Lambda-\lambda_{1}\right)^{3} M_{1}^{(3)}(\Lambda) w=w$, and therefore the solution of (9) in this case can be written in the form

$$
U(x)=\frac{1}{c} A^{*}(\Lambda) M_{1}^{(3)}(\Lambda) V(x) .
$$

Next, we find out conditions which must be imposed on the function $V(x)$, so we can use formulas (12), (14) and (15). First, the following limits must be true $\lim _{t \rightarrow 0} t^{-\lambda_{i}} V(t x)=0$, where $i=1,2,3$ for the different roots, or $\lim _{t \rightarrow 0} \ln ^{k} t t^{-\lambda_{1}} V(t x)=0(k=1,2)$ in the case of multiple roots. Second, to ensure that the function $U(x)$ has the necessary smoothness to perform the boundary conditions of the problem, it is necessary that the operator $A^{*}(\Lambda)$ is applicable to the function $V(x)$ in $\bar{S}$. Due to the structure of the operator $A^{*}(\Lambda)$ it is possible if $v_{1} \in C^{2}(\bar{S})$ and $v_{2} \in C^{1}(\bar{S})$. Such smoothness of the functions $v_{1}(x)$ and $v_{2}(x)$ is provided if $\varphi_{1} \in C^{2}(\partial S)$ and $\varphi_{2} \in C^{1}(\partial S)$. This is accomplished.

Let us return to the conditions

$$
\lim _{t \rightarrow 0} t^{-\lambda_{i}} V(t x)=0, \quad i=1,2,3,
$$


which in fact are imposed on the functions $\varphi_{1} \in C^{2}(\partial S)$ and $\varphi_{2} \in C^{1}(\partial S)$. Note that due to the smoothness of the functions $V(x)$, for $\lambda_{i} \notin \mathbb{N}_{0}$ conditions $\lim _{t \rightarrow 0} t^{-\lambda_{i}} V(t x)=0$ and $\lim _{t \rightarrow 0} \ln ^{k} t t^{-\lambda_{i}} V(t x)=0$ are equivalent. If $\lambda_{i}<0$, then conditions (16) are fulfilled. Otherwise, i.e. if at least for one $i$ we have $\lambda_{i}>0$, then (16) can be not fulfilled for some functions $\varphi_{1}$ and $\varphi_{2}$.

Let all the roots of $\lambda_{i}$ be different. Change the form of the solution (12) in case $\lambda_{i}>0$. At first note that if $\lim _{t \rightarrow \infty} t^{-\lambda_{i}} w(t x)=0$, then for the operator

$$
\widehat{M}_{i}(\Lambda) v(x)=-\int_{1}^{\infty} t^{-\lambda_{i}-1} v(t x) d t
$$

where $w(x) \in C^{1}\left(\mathbb{R}^{n}\right)$, the equalities similar to (10) are true

$$
\begin{gathered}
\left(\Lambda-\lambda_{i}\right) \widehat{M}_{i}(\Lambda) w=\lambda_{i} \int_{1}^{\infty} t^{-\lambda_{i}-1} w(t x) d t-\int_{1}^{\infty} t^{-\lambda_{i}} w_{t}(t x) d t=\lambda_{i} \int_{1}^{\infty} t^{-\lambda_{i}-1} w(t x) d t- \\
-\left.t^{-\lambda_{i}} w(t x)\right|_{1} ^{\infty}-\lambda_{i} \int_{1}^{\infty} t^{-\lambda_{i}-1} w(t x) d t=-\left.t^{-\lambda_{i}} w(t x)\right|_{1} ^{\infty}=w(x) .
\end{gathered}
$$

For example, if $|\alpha|<\lambda_{i}$ then we have

$$
\widehat{M}_{i}(\Lambda) x^{\alpha}=-x^{\alpha} \int_{1}^{\infty} t^{|\alpha|-\lambda_{i}-1} d t=-\left.x^{\alpha} \frac{t^{|\alpha|-\lambda_{i}}}{|\alpha|-\lambda_{i}}\right|_{1} ^{\infty}=\frac{x^{\alpha}}{|\alpha|-\lambda_{i}},
$$

where $x^{\alpha}=x_{1}^{\alpha_{1}} \ldots x_{n}^{\alpha_{n}}$ и $|\alpha|=\alpha_{1}+\cdots+\alpha_{n}$, i.e. the operator $\widehat{M}_{i}(\Lambda)$ is applicable to the polynomial $x^{\alpha}$. Change formula (12). Let $n_{i}=\left[\lambda_{i}\right]$. We represent the function $V(x)$ in the form

$$
V(x)=\sum_{k=0}^{n_{i}} \sum_{j=1}^{h_{k}} V_{k}^{(j)} H_{k}^{(j)}(x)+\left(V(x)-\sum_{k=0}^{n_{i}} \sum_{j=1}^{h_{k}} V_{k}^{(j)} H_{k}^{(j)}(x)\right) \equiv V_{1}^{(i)}(x)+V_{2}^{(i)}(x),
$$

where $\left\{H_{m}^{(i)}(x), m \in \mathbb{N}_{0}, i=\overline{1, h_{k}}\right\}$ is a complete orthogonal system of homogeneous harmonic polynomials on $\partial S$ [15], and $V_{k}^{(j)}$ are expansion coefficients of the function $V(x)$ by this system. It is clear that if $n_{i}=\left[\lambda_{i}\right]<0$ then $V_{1}^{(i)}(x)=0$ and thus $V_{2}^{(i)}(x)=V(x)$. Consider the expression

$$
U_{i}(x)=b_{i} A^{*}(\Lambda)\left(\widehat{M}_{i}(\Lambda) V_{1}^{(i)}(x)+M_{i}(\Lambda) V_{2}^{(i)}(x)\right) .
$$

Since $V_{1}^{(i)}(x)$ is a polynomial of degree $n_{i}$, then for each its term of the form $V_{k}^{(j)} H_{k}^{(j)}(x)$ we have $k-\lambda_{i} \leq n_{i}-\lambda_{i}=\left[\lambda_{i}\right]-\lambda_{i}<0$ (no equals sign, because the numbers $\lambda_{i}$ are not integer) and hence by virtue of (17) the operator $\widehat{M}_{i}(\Lambda)$ is applicable to the polynomial $V_{1}^{(i)}(x)$. Hence $-\lambda_{i}+n_{0}+1=-\lambda_{i}+\left[\lambda_{i}\right]+1>0$, then $t^{-\lambda_{i}} V_{2}^{(i)}(t x) \sim t^{-\lambda_{i}+n_{0}+1} V_{2}^{(i)}(x) \rightarrow 0$ as $t \rightarrow 0$. So, the operator $M_{i}(\Lambda)$ is applicable to the function $V_{2}^{(i)}(x)$. Thus expression (18) makes sense.

By properties of the operators $\widehat{M}_{i}(\Lambda)$ and $M_{i}(\Lambda)$ the following equalities hold

$$
A(\Lambda) U_{i}(x)=b_{i} A(\Lambda) A^{*}(\Lambda)\left(\widehat{M}_{i}(\Lambda) V_{1}^{(i)}(x)+M_{i}(\Lambda) V_{2}^{(i)}(x)\right)=
$$




$$
\begin{gathered}
=b_{i} \Delta(\Lambda)\left(\widehat{M}_{i}(\Lambda) V_{1}^{(i)}(x)+M_{i}(\Lambda) V_{2}^{(i)}(x)\right)= \\
=c b_{i}\left(\Lambda-\lambda_{2}\right)\left(\Lambda-\lambda_{3}\right)\left(V_{1}^{(i)}(x)+V_{2}^{(i)}(x)\right)=c b_{i}\left(\Lambda-\lambda_{2}\right)\left(\Lambda-\lambda_{3}\right) V .
\end{gathered}
$$

It means that the following function

$$
U(x)=U_{1}(x)+U_{2}(x)+U_{3}(x)
$$

satisfies the following equality

$$
\begin{gathered}
A(\Lambda) U=\sum_{i=1}^{3} A(\Lambda) U_{i}(x)=\left(c b_{1}\left(\Lambda-\lambda_{2}\right)\left(\Lambda-\lambda_{3}\right)+c b_{2}\left(\Lambda-\lambda_{1}\right)\left(\Lambda-\lambda_{3}\right)+\right. \\
\left.+c b_{3}\left(\Lambda-\lambda_{1}\right)\left(\Lambda-\lambda_{2}\right)\right) V=1 \cdot V=V .
\end{gathered}
$$

This means that the function $U(x)$ from (19) is a solution of $(9)$ in $\bar{S}$. Therefore the function $u(x)=u_{0}(x)+|x|^{2} u_{1}(x)$ is a solution of $(5)-(6)$.

In the case of duple roots $\lambda_{1}=\lambda_{2}>0$ together with the operator $\widehat{M}_{1}(\Lambda)$ it is necessary to consider the operator

$$
\widehat{M}_{1}^{(2)}(\Lambda) v(x)=\int_{1}^{\infty} \ln t t^{-\lambda_{i}-1} v(t x) d t
$$

for which in the case of a differentiable function $w(x) \in C^{1}\left(\mathbb{R}^{n}\right)$ the equalities hold

$$
\left(\Lambda-\lambda_{1}\right) \widehat{M}_{1}^{(2)}(\Lambda) w=-\left.t^{-\lambda_{i}} w(t x)\right|_{1} ^{\infty}+\widehat{M}_{1}(\Lambda) w=\widehat{M}_{1}(\Lambda) w(x)
$$

and thus $\left(\Lambda-\lambda_{1}\right)^{2} \widehat{M}_{1}^{(2)}(\Lambda) w=w$. For example, when $|\alpha|<\lambda_{1}$ we have

$$
\begin{aligned}
\widehat{M}_{1}^{(2)}(\Lambda) x^{\alpha} & =x^{\alpha} \int_{1}^{\infty} \ln t t^{|\alpha|-\lambda_{1}-1} d t=\frac{x^{\alpha}}{|\alpha|-\lambda_{1}} \int_{1}^{\infty} \ln t d t^{|\alpha|-\lambda_{1}}=\left.x^{\alpha} \frac{t^{|\alpha|-\lambda_{1}} \ln t}{|\alpha|-\lambda_{1}}\right|_{1} ^{\infty}- \\
& -\frac{x^{\alpha}}{|\alpha|-\lambda_{1}} \int_{1}^{\infty} t^{|\alpha|-\lambda_{1}-1} d t=\frac{1}{|\alpha|-\lambda_{1}} \widehat{M}_{1}(\Lambda) x^{\alpha}=\frac{1}{\left(|\alpha|-\lambda_{1}\right)^{2}}
\end{aligned}
$$

and hence the operator $\widehat{M}_{1}^{(2)}(\Lambda)$ is applicable to the polynomial $V_{1}^{(1)}(x)$. The solution of (9) can be represented in the form

$$
U(x)=U_{1}(x)+U_{1}^{(2)}(x)+U_{3}(x),
$$

where the functions $U_{1}(x)$ and $U_{3}(x)$ are defined in (18), and the function $U_{1}^{(2)}(x)$ is written in the form

$$
U_{1}^{(2)}(x)=b_{2} A^{*}(\Lambda)\left(\widehat{M}_{1}^{(2)}(\Lambda) V_{1}^{(1)}(x)+M_{1}^{(2)}(\Lambda) V_{2}^{(1)}(x)\right)
$$

In the case of triple roots $\lambda_{1}=\lambda_{2}=\lambda_{3}>0$ the solution of (9) has the form

$$
U(x)=\frac{1}{c} A^{*}(\Lambda)\left(\widehat{M}_{1}^{(3)}(\Lambda) V_{1}^{(1)}(x)+M_{1}^{(3)}(\Lambda) V_{2}^{(1)}(x)\right),
$$

where $\widehat{M}_{1}^{(3)}(\Lambda) v(x)=-\frac{1}{2} \int_{0}^{1} \ln ^{2} t t^{-\lambda_{1}-1} v(t x) d t$, and the operator $M_{1}^{(3)}(\Lambda)$, the functions $V_{1}^{(1)}(x)$ and $V_{2}^{(1)}(x)$ are defined as above. Thus, the solution of (9) in $\bar{S}$ is constructed in all cases of the roots of polynomial $\Delta(\lambda)$. After finding the solution of (9) that are harmonic functions $u_{0}(x)$ and $u_{1}(x)$, a solution of $(5)-(6)$ can be written in the form $u(x)=u_{0}(x)+|x|^{2} u_{1}(x)$. 


\section{Existence of Conditional Solutions}

Consider the case of problem (5) - (6) which is not investigated in Theorem 2, when for some $m \in \mathbb{N}_{0}$ the equality $\Delta(m)=0$ holds. Problem (1) - (2) with nonhomogeneous biharmonic equation is considered in [18].

Theorem 3. Let for some $m \in \mathbb{N}_{0}$ the equality $\Delta(m)=0$ hold. Then a solution of (5) - (6) from the class $u \in C^{3}(\bar{S})$ exists if and only if the functions $\varphi_{1} \in C^{2}(\partial S)$ and $\varphi_{2} \in C^{1}(\partial S)$ satisfy the equalities

$$
\int_{\partial S} H_{m}(x)\left(q_{1}(m) \varphi_{1}(x)+q_{2}(m) \varphi_{2}(x)\right) d s_{x}=0,
$$

where $H_{m}(x)$ is an arbitrary homogeneous harmonic polynomial of degree $m$, and the vector $\left(\begin{array}{l}q_{1}(m) \\ q_{2}(m)\end{array}\right)$ is an arbitrary solution of the system of algebraic equations

$$
\begin{aligned}
& \left(\begin{array}{cc}
a_{00}+a_{01} m & a_{11} m \\
a_{00}+(m+2) a_{01}+(2 n+4 m) a_{02} & (m+2) a_{11}+(2 n+4 m)\left(a_{12}+m a_{13}\right)
\end{array}\right) \times \\
& \times\left(\begin{array}{c}
q_{1}(m) \\
q_{2}(m)
\end{array}\right)=0 .
\end{aligned}
$$

Proof. Solution of problem (5) - (6) constructed in Theorem 2 under the conditions of the present theorem is not suitable. It must be a little changed. Consider equation (9). We expand the function $V(x)$ onto two terms $V(x)=\widehat{V}(x)+V_{m}(x)$, where

$$
V_{m}(x)=\sum_{i=1}^{h_{m}} P_{m}^{(i)} H_{m}^{(i)}(x)
$$

We find conditions under which (9) has a solution in a particular case, when the right-hand side is a monomial of the form $V_{m}(x)=P_{m}^{(i)} H_{m}^{(i)}(x)$, where

$$
P_{m}^{(i)}=\frac{1}{\left\|H_{m}^{(i)}\right\|_{L_{2}(\partial S)}^{2}}\left(\begin{array}{c}
\int_{\partial S} H_{m}^{(i)}(x) \varphi_{1}(x) d s_{x} \\
\int_{\partial S} H_{m}^{(i)}(x) \varphi_{2}(x) d s_{x}
\end{array}\right) .
$$

By the homogeneity of the operator $A(\Lambda)$ the solution of (9) in this case may have only the form $U_{m}(x)=Q_{m}^{(i)} H_{m}^{(i)}(x)$. Substituting the polynomial $U_{m}(x)=Q_{m}^{(i)} H_{m}^{(i)}(x)$ to (9) we have

$$
A(\Lambda) Q_{m}^{(i)} H_{m}^{(i)}(x)=H_{m}^{(i)}(x) A(m) Q_{m}^{(i)}=H_{m}^{(i)}(x) P_{m}^{(i)},
$$

and consequently the following equality should be true

$$
A(m) Q_{m}^{(i)}=P_{m}^{(i)} .
$$

It is known that the obtained system of algebraic equations has a solution only if the right-hand side $P_{m}^{(i)}$ is orthogonal to zeros of the conjugated system $A^{T}(m) Q=0$, i.e. to all vectors $\left(\begin{array}{c}q_{1}(m) \\ q_{2}(m)\end{array}\right)$ from the theorem's conditions

$$
P_{m}^{(i)} \cdot\left(\begin{array}{c}
q_{1}(m) \\
q_{2}(m)
\end{array}\right)=\left(\begin{array}{l}
\int_{\partial S} H_{m}^{(i)}(x) \varphi_{1}(x) d s_{x} \\
\int_{\partial S} H_{m}^{(i)}(x) \varphi_{2}(x) d s_{x}
\end{array}\right) \cdot\left(\begin{array}{c}
q_{1}(m) \\
q_{2}(m)
\end{array}\right)=\int_{\partial S} q_{1}(m) H_{m}^{(i)}(x) \varphi_{1}(x) d s_{x}+
$$




$$
+\int_{\partial S} q_{2}(m) H_{m}^{(i)}(x) \varphi_{2}(x) d s_{x}=\int_{\partial S} H_{m}^{(i)}(x)\left(q_{1}(m) \varphi_{1}(x)+q_{2}(m) \varphi_{2}(x)\right) d s_{x}=0 .
$$

By virtue of (22) this condition is satisfied. Therefore a solution of (25) exists. Going through the all terms in sum (24) and taking advantage of the theorem's conditions, we conclude that a solution of (9) for $V(x)=V_{m}(x)$ exists. We denote it as $U_{m}(x)$. After that, if the polynomial $\Delta(\lambda)$ has no other roots in $\mathbb{N}_{0}$, then by Theorem 2 we solve equation (9) for $V(x)=\widehat{V}(x)$ and add the resulting solutions $U(x)=U_{m}(x)+\widehat{U}(x)$. The function $U(x)$ is a solution of $(9)$ in $\bar{S}$, and hence problem (5) - (6) has a solution. If the polynomial $\Delta(\lambda)$ has also other roots in $\mathbb{N}_{0}$ except number $m$, then we proceed with the function $\widehat{V}(x)$ in a similar way, as we did above with the function $V(x)$. Thus, for each root of the polynomial $\Delta(\lambda)$ from $\mathbb{N}_{0}$ conditions (22) must be satisfied.

\section{Particular Cases of the Problem}

1. Riquier - Neumann problem: let $a_{01} \neq 0, a_{13} \neq 0$ (all other coefficients are equal to zero), then

$$
\Delta^{2} u=0, x \in S ;\left.\quad a_{01} \frac{\partial}{\partial \nu} u\right|_{\partial S}=\varphi_{1}(s),\left.a_{13} \frac{\partial}{\partial \nu} \Delta u\right|_{\partial S}=\varphi_{2}(s), \quad s \in \partial S .
$$

The uniqueness conditions of this problem have the form $\Delta(\lambda)=\lambda^{2}(2 n+4 \lambda) a_{01} a_{13}$ and hence $\Delta(0)=0$. The algebraic system (3) has the form $\left(\begin{array}{cc}0 & 2 a_{01} \\ 0 & 0\end{array}\right)\left(\begin{array}{l}C_{1} \\ C_{2}\end{array}\right)=0$, and its solution can be written in the form $C_{2}=0, C_{1}$ is an arbitrary constant. By Theorem 2 solution of (26) is unique up to a constant $u(x)=C_{1} H_{0}(x)=C_{1}$.

For the existence of solutions of (26) consider (23) for $m=0,\left(\begin{array}{cc}0 & 0 \\ 2 a_{01} & 0\end{array}\right)\left(\begin{array}{l}q_{1}(0) \\ q_{2}(0)\end{array}\right)=0$, and hence $q_{1}(0)=0$. In this case the space of zeros of system (23) is one-dimensional and is spanned by the vector $\left(\begin{array}{l}0 \\ 1\end{array}\right)$. Therefore, the existence condition (22) of solutions of $(26)$ has the form $\int_{\partial S} \varphi_{2}(x) d s_{x}=0$. For example, let $a_{01}=a_{13}=1$ and $\varphi_{1}=0, \varphi_{2}=1$. The obtained above existence condition for the problem's solution is not fulfilled. Make sure that a solution of (26) in this case does not exist. From the second boundary condition of problem (26) follows that for solution $u(x)=u_{0}(x)+|x|^{2} u_{1}(x)$ (see Lemma 1) of this problem the following equalities must be fulfilled

$$
\omega_{n}=\int_{\partial S} d s_{x}=\int_{\partial S} \frac{\partial}{\partial \nu}(2 n+4 \Lambda) u_{1}(x) d s_{x}=4 \int_{\partial S} \frac{\partial^{2}}{\partial \nu^{2}} u_{1}(x) d s_{x}=0
$$

which is not true. Here it was taken into account that the integral on $\partial S$ of the normal derivatives of harmonic in $S$ functions is equal to zero (see [17]). Therefore (26) has no solution.

2. For problem (5) - (6) if $a_{02}=0$ and $a_{11}=0$ we have

$\Delta(\lambda)=\left|\begin{array}{cc}a_{00}+a_{01} \lambda & 2 a_{01} \\ 0 & (2 n+4 \lambda) a_{12}+\lambda(2 n+4 \lambda) a_{13}\end{array}\right|=(2 n+4 \lambda)\left(a_{00}+\lambda a_{01}\right)\left(a_{12}+\lambda a_{13}\right)$. 
Let us take in (2) $a_{00}=-2, a_{01}=1, a_{12}=-3, a_{13}=1$, i.e. consider the problem

$$
\begin{gathered}
\Delta^{2} u=0, x \in S \\
-2 u+\left.\frac{\partial u}{\partial \nu}\right|_{\partial S}=\varphi_{1}(s),-3 \Delta u+\left.\frac{\partial \Delta u}{\partial \nu}\right|_{\partial S}=\varphi_{2}(s), s \in \partial S .
\end{gathered}
$$

In this case $\Delta(\lambda)=(2 n+4 \lambda)(\lambda-2)(\lambda-3)$ and hence $\Delta(2)=0$ and $\Delta(3)=0$.

For $m=2$ system (3) has the form $\left(\begin{array}{cc}0 & 2 \\ 0 & -(2 n+8)\end{array}\right)\left(\begin{array}{l}C_{1} \\ C_{2}\end{array}\right)=0$ and therefore $C_{2}=$ $0, C_{1}$ being an arbitrary constant, is its solution. Thus the polynomial $u_{2}(x)=C_{1} H_{2}(x)$ is a solution of the homogeneous problem (27).

If $m=3$, then the system of (3) takes the form $\left(\begin{array}{ll}1 & 2 \\ 0 & 0\end{array}\right)\left(\begin{array}{l}C_{1} \\ C_{2}\end{array}\right)=0$ and hence $C_{1}=-2 C_{2}, C_{2}$ being an arbitrary constant, is its solution. Hence the polynomial $u_{3}(x)=$ $C_{2}\left(|x|^{2}-3\right) H_{3}(x)$ is also a solution of the homogeneous problem (27).

For the existence of solutions of (27) consider system (23)

$$
\left(\begin{array}{cc}
m-2 & 0 \\
m & (m-3)(2 n+4 m)
\end{array}\right)\left(\begin{array}{l}
q_{1}(m) \\
q_{2}(m)
\end{array}\right)=0
$$

for $m=2$ and $m=3$. Let $m=2$, then we have $\left(\begin{array}{cc}0 & 0 \\ 2 & -(2 n+8)\end{array}\right)\left(\begin{array}{l}q_{1}(2) \\ q_{2}(2)\end{array}\right)=0$, and hence $q_{1}(2)-(n+4) q_{2}(2)=0$. In this case the space of zeros of $(23)$ is one-dimensional and is spanned by the vector $\left(\begin{array}{c}n+4 \\ 1\end{array}\right)$. So, the first condition of existence of solutions to $(27)$ has the form $\int_{\partial S} H_{2}(x)\left((n+4) \varphi_{1}(x)+\varphi_{2}(x)\right) d s_{x}=0$.

Let $m=3$, then we obtain $\left(\begin{array}{ll}1 & 0 \\ 3 & 0\end{array}\right)\left(\begin{array}{l}q_{1}(3) \\ q_{2}(3)\end{array}\right)=0$, and hence $q_{1}(3)=0$. In this case the space of zeros of $(23)$ is also one-dimensional and is spanned by the vector $\left(\begin{array}{l}0 \\ 1\end{array}\right)$. So the second condition of existence of solutions to (27) has the form $\int_{\partial S} H_{3}(x) \varphi_{2}(x) d s_{x}=0$. Thus, (27) has a solution if

$$
\int_{\partial S} H_{2}(x)\left((n+4) \varphi_{1}(x)+\varphi_{2}(x)\right) d s_{x}=0, \quad \int_{\partial S} H_{3}(x) \varphi_{2}(x) d s_{x}=0,
$$

where $H_{2}(x)$ and $H_{3}(x)$ are homogeneous harmonic polynomials of degrees 2 and 3.

For example, let $n \geq 2, \varphi_{1}=s_{1} s_{2}$ and $\varphi_{2}=0$. If a solution of (27) exists, then it takes the form $u(x)=u_{0}(x)+|x|^{2} u_{1}(x)$ (see Lemma 1). From the second boundary condition we have $(2 n+4 \Lambda)(\Lambda-3) u_{1}(x)=0$, whence $(\Lambda-3) u_{1}(x)=0$ and therefore $u_{1}(x)=H_{3}(x)$. By virtue of the first boundary condition the function $u_{0}(x)$ should be such that $\left.\left(\frac{\partial}{\partial \nu}-2\right)\left(u_{0}(x)+|x|^{2} H_{3}(x)\right)\right|_{\partial S}=s_{1} s_{2}$ and hence $(\Lambda-2) u_{0}(x)=x_{1} x_{2}-3 H_{3}(x)$, $x \in S$. This equation has no solution in harmonic functions in $S$ because a harmonic function on the left-hand side $(\Lambda-2) u_{0}(x)$ does not contain the second order terms in its expansion at the original, but on the right-hand side such terms are present. Note that in this case the first condition from (28) is not fulfilled for $H_{2}(x)=x_{1} x_{2}$ because $\int_{\partial S} x_{1}^{2} x_{2}^{2} d s_{x} \neq 0$. 
Acknowledgements. The second named author is financially supported by a grant from the Ministry of Science and Education of the Republic of Kazakhstan (Grant No. 0819/GF4).

\section{References / Литература}

1. Andersson L-E., Elfving T., Golub G.H. Solution of Biharmonic Equations with Application to Radar Imaging. Journal of Computational and Applied Mathematics, 1998, vol. 94, no. 2, pp. 153-180. DOI: 10.1016/S0377-0427(98)00079-X

2. Lai M.-C., Liu H.-C. Fast Direct Solver for the Biharmonic Equation on a Disk and Its Application to Incompressible Flows. Applied Mathematics and Computation, 2005, vol. 164, no. 3, pp. 679-695. DOI: $10.1016 /$ j.amc.2004.04.064

3. Ehrlich L.N., Gupta M.M. Some Difference Schemes for the Biharmonic Equation. SIAM Journal on Numerical Analysis, 1975, vol. 12, no. 5, pp. 773-790. DOI: 10.1137/0712058

4. Almansi E. Sull'integrazione dell'equazione differenziale $\Delta^{2 n} u=0$. Annali di Matematica Pura ed Applicata, 1899, vol. 2, no. 3, pp. 1-51. DOI: 10.1007/BF02419286

5. Boggio T. Sulle funzioni di green d'ordinem. Rendiconti del Circolo Matematico di Palermo, 1905, pp. $97-135$.

6. Love A.E.H. Biharmonic Analysis, Especially in a Rectangle, and Its Application to the Theory of Elasticity. Journal London Mathematical Society, 1928. vol. 3, pp. 144-156. DOI: $10.1112 / \mathrm{jlms} / \mathrm{s} 1-3.2 .144$

7. Zaremba S. Sur l'integration de l'equation biharmonique. Bulletin International de l'Academie des Sciences de Cracovie, 1908, pp. 1-29.

8. Karachik V.V. Construction of Polynomial Solutions to the Dirichlet Problem for the Polyharmonic Equation in a Ball. Computational Mathematics and Mathematical Physics, 2014, vol. 54, no. 7, pp. 1122-1143. DOI: 10.1134/S0965542514070070 [Карачик, В.В. Построение полиномиальных решений задачи Дирихле для полигармонического уравнения в шаре / В.В. Карачик // Журнал вычислительной математики и математической физики. - 2014. - Т. 54, № 7. - С. 1149-1170.]

9. Karachik V.V. Normalized System of Functions with Respect to the Laplace Operator and Its Applications. Journal of Mathematical Analysis and Applications, 2003, vol. 287, no. 2, pp. 577-592. DOI: 10.1016/S0022-247X(03)00583-3

10. Karachik V.V., Turmetov B.Kh., Bekaeva A. Solvability Conditions of the Neumann Boundary Value Problem for the Biharmonic Equation in the Unit Ball. International Journal of Pure and Applied Mathematics, 2012. vol. 81, no. 3, pp. 487-495.

11. Karachik V.V. Solvability Conditions for the Neumann Problem for the Homogeneous Polyharmonic Equation. Differential Equations, 2014, vol. 50, no. 11, pp. 1449-1456. DOI: 10.1134/S0012266114110032 [Карачик, B.В. Условия разрешимости задачи Неймана для однородного полигармонического уравнения / В.В. Карачик // Дифференциальные уравнения. - 2014. - Т. 50, № 11. - С. 1455-1461.]

12. Karachik V.V. On Solvability Conditions for the Neumann Problem for a Polyharmonic Equation in the Unit Ball. Journal of Applied and Industrial Mathematics, 2014, vol. 8, no. 1, pp. 63-75. DOI: 10.1134/S1990478914010074 [Карачик, B.В. Об условиях разрешимости задачи Неймана для полигармонического уравнения в единичном шаре / В.В. Карачик // Сибирский журнал индустриальной математики. - 2013. - Т. 16, № 4 (56). - С. 61-74.]

13. Gazzola F., Sweers G. On Positivity for the Biharmonic Operator under Steklov Boundary Conditions. Archive for Rational Mechanics and Analysis, 2008, vol. 188, pp. 399-427. DOI: $10.1007 / \mathrm{s} 00205-007-0090-4$ 
14. Karachik V.V., Sadybekov M.A., Torebek B.T. Uniqueness of Solutions to Boundary-Value Problems for the Biharmonic Equation in a Ball. Electronic Journal of Differential Equations, 2015, vol. 2015, no. 244, pp. 1-9.

15. Karachik V.V. Construction of Polynomial Solutions to Some Boundary Value Problems for Poisson's Equation. Computational Mathematics and Mathematical Physics, 2011. vol. 51, no. 9, pp. 1567-1587. DOI: 10.1134/S0965542511090120 [Карачик, В.В. Построение полиномиальных решений некоторых краевых задач для уравнения Пуассона / B.B. Kapaчик // Журнал вычислительной математики и математической физики. - 2011. - Т. 51, № 9. - C. 1674-1694.]

16. Karachik V.V. A Problem for the Polyharmonic Equation in the Sphere. Siberian Mathematical Journal, 1991. vol. 32, no. 5, pp. 767-774. DOI: 10.1007/BF00971175 [Kapaчик, В.В. Об одной задаче для полигармонического уравнения в шаре / В.В. Карачик // Сибирский математический журнал. - 1991. - Т. 32, № 5. - С. 51-58.]

17. Karachik V.V. On the Mean Value Property for Polyharmonic Functions in the Ball. Siberian Advances in Mathematics, 2014, vol. 24, no. 3, pp. 169-182. DOI: 10.3103/S1055134414030031 [Карачик, В.В. О свойстве среднего для полигармонических функций в шаре / В.В. Карачик // Математические труды. - 2013. - Т. 16, № 2. - С. 69-88.]

18. Karachik V.V., Torebek B.T. On Uniqueness and Correct Solvability of the Biharmonic Boundary Value Problem. AIP Conference Proceedings, 2016, vol. 1759, 020045, 4 p. DOI: $10.1063 / 1.4959659$

Received September 26, 2016

УДК 517.9

DOI: $10.14529 / \mathrm{mmp} 160404$

\section{ОБ ОДНОЙ МАТЕМАТИЧЕСКОЙ МОДЕЛИ, ОПИСЫВАЕМОЙ КРАЕВОЙ ЗАДАЧЕЙ ДЛЯ БИГАРМОНИЧЕСКОГО УРАВНЕНИЯ}

\section{В.В. Карачик, Б.Т. Торебек}

В данной работе рассматривается математическая модель, описываемая обобщенной третьей краевой задачи для однородного бигармонического уравнения в шаре с граничными операторами до третьего порядка, содержащие нормальные производные и лапласиан. Частными случаями рассматриваемой математической модели являются классические модели, описываемые задачами Дирихле, Рикье и Робина, спектральная задача Стеклова, а также многие другие математические модели, порожденные этими граничными условиями. Доказаны две теоремы существования рассматриваемой задачи. Условия существования получены в виде ортогональности на границе некоторой линейной комбинации граничных функций однородным гармоническим многочленам заданного порядка. Полученные результаты проиллюстрированы некоторыми частными случаями общей задачи.

Ключевые слова: математическая модель; бигармоническое уравнение; граничная задача; оператор Лапласа.

Валерий Валентинович Карачик, доктор физико-математических наук, профессор, кафедра математического анализа и методики преподавания математики, Южно-Уральский государственный университет (г. Челябинск, Российская Федерация), karachik@susu.ru.

Берикбол Тиллабайулы Торебек, докторант, Институт математики и математического моделирования (г. Алматы, Республика Казахстан), torebek@math.kz.

Поступила в редакиию 26 сентября 2016 г. 